\title{
Feasibility of a supported self-management intervention for relatives of people with recent-onset psychosis: REACT study
}

Fiona Lobban, David Glentworth, Lesley Chapman, Laura Wainwright, Adam Postlethwaite, Graham Dunn, Vanessa Pinfold, Warren Larkin and Gillian Haddock

\section{Background}

Relatives of people with psychosis experience high levels of distress and require support. Family interventions have been shown to be effective in improving outcomes but are difficult to access and not suitable for all relatives.

\section{Aims \\ To assess the feasibility and effectiveness of a supported self-management package for relatives of people with recent-onset psychosis.}

\section{Method}

A randomised controlled trial $(n=103)$ comparing treatment as usual (TAU) in early intervention services with TAU plus the Relatives' Education And Coping Toolkit (REACT) intervention (trial identifier: ISRCTN69299093).

\section{Results}

Compared with TAU only, those receiving the additional REACT intervention showed reduced distress and increased perceived support and perceived ability to cope at 6-month follow-up.

\section{Conclusions}

The toolkit is a feasible and potentially effective intervention to improve outcomes for relatives. A larger trial is needed to reliably assess the clinical and cost-effectiveness of REACT, and its impact on longer-term outcomes.

\section{Declaration of interest}

None.
First-episode psychosis commonly occurs in adolescence when most people live with their parents. It is associated with high levels of distress in relatives ${ }^{1,2}$ evident from a very early stage of illness. ${ }^{3,4}$ The UK government has expressed a strong commitment to supporting relatives, ${ }^{5}$ not least because the care they give saves the National Health Service (NHS) a vast amount of money each year. ${ }^{6}$ Family interventions have been shown to be effective in improving outcomes for people with schizophrenia ${ }^{7}$ and are recommended by the National Institute for Health and Care Excellence (NICE). ${ }^{8}$ One meta-analysis suggests positive benefits from family interventions in first-episode populations, ${ }^{9}$ although this approach may not always be suitable at this stage. ${ }^{10}$ The impact of family interventions on relatives' outcomes such as distress and well-being is less clear as often these are either not assessed or only as secondary outcomes. ${ }^{11}$ Implementation of family interventions in real-world clinical settings is poor. Significant barriers include lack of engagement with families, lack of confidence and training among clinicians, high case-loads, and an individualised model of care. ${ }^{12}$ Consistent with government policy on the management of long-term conditions ${ }^{13}$ is the option of developing supported self-management approaches that provide relatives with the knowledge and skills they need, at a pace and intensity they can control. Such approaches have the potential to vastly improve access, are inherently empowering in their ethos and are relatively easy and cheap to deliver. Although self-management approaches are being developed across a wide range of physical health conditions, ${ }^{14}$ less progress has been made in mental health, with the notable exception of eating disorders. ${ }^{15,16}$ The Relatives' Education And Coping Toolkit (REACT) study is the first study to evaluate the feasibility and effectiveness of a supported self-management intervention for relatives of people experiencing recent-onset psychosis (trial identifier: ISRCTN69299093). The main aims were to determine: (a) the acceptability of the intervention; (b) preference for type of support; (c) rates of recruitment and retention; and (d) the likely effect size on a range of outcomes for relatives.

\section{Method}

This study was approved by a local NHS ethics committee (REC ref: 08/H1001/147) and three participating NHS trusts in North West England.

\section{Trial design}

This is a stratified randomised controlled trial in which participants were allocated to receive either treatment as usual (TAU) or TAU plus the REACT intervention. Following baseline assessments, independent randomisation was done by a UK clinical trials unit using permuted blocks within NHS trust with randomly varying block sizes. A detailed study protocol is published elsewhere. ${ }^{17}$

\section{Participants}

Participants were relatives, partners or close friends of people experiencing psychosis who were currently being supported by the early intervention services within a participating NHS trust. These teams support young people experiencing psychotic symptoms between the ages of 14 and 35 for a period of up to 3 years. ${ }^{18}$ Only relatives in direct contact with early intervention services were invited to take part. Only one relative per family (the self-identified main carer) took part in the study but they were encouraged to share the intervention with other close family members. Additional inclusion criteria were that the first contact with early intervention services was within the past 24 months and that participants were aged over 18 and had sufficient understanding of written and spoken English (translations and interpreters were not routinely available). All participants gave written informed consent. All service users were informed about the study but were not required to consent as they were not participants. 


\section{Procedure}

Assessments were carried out in face-to-face interviews in the relatives' home, NHS or university premises at baseline and 6-month follow-up by a research assistant who was masked to allocation. Relatives in both arms of the study were invited to take part in qualitative interviews about the difficulties they faced in supporting someone with psychosis, and how they coped with these. Those in the REACT group also gave feedback on the intervention. These data will be reported elsewhere.

\section{The REACT intervention}

The development of the REACT intervention was informed by (a) a systematic review of interventions reporting on outcomes for relatives of people with psychosis; ${ }^{11}$ (b) a series of focus groups with relatives; ${ }^{19}$ (c) clinical and personal expertise within the research team (consisting of relatives, clinicians and academics); and (d) extensive feedback from service users and relatives throughout the process.

The toolkit is comprehensive and modular in format so that the content is divided into 13 manageable sections that can be used flexibly depending on the individual needs of the relative. These include: introduction to REACT; what is psychosis?; managing positive symptoms; managing negative symptoms; dealing with crises; dealing with difficult behaviour; managing stress - thinking differently; managing stress - doing things differently; understanding mental health services; treatment options; the future; resource directory; jargon terms. Modules range between 11 and 23 A5 pages, although the resource directory is considerably longer at 43 pages. Extensive use is made of signposting to other resources, which means the toolkit needs to be updated regularly. Although the information is about psychosis in general, the toolkit is designed to help relatives to make this information specific to their family by identifying key questions they may need to ask to get the information they require. Case examples are used to aid illustration. The content of the toolkit reflects the key ingredients in existing evidencebased family interventions. All participants were given a printed version of the toolkit and offered an online version.

Each participant was supported in the use of the toolkit by an early intervention services support worker. These workers are well placed to support this self-management approach as it does not require highly trained health professionals, but does require availability and flexibility. They are also relatively inexpensive, lowering cost barriers to further dissemination. Supporters attended 4 days of training spaced over 3 months, followed by monthly group supervision for the duration of the project. The early intervention services in the three NHS trusts were organised in a hub and spoke model and further divided into smaller teams to cover the wide geographical areas. One trust identified a supporter to be trained in each of two teams and two supporters in the third team. Another trust allocated one supporter to work across all three of its teams. The final trust operated as a single team and had one supporter. In total seven teams took part and six supporters were trained.

All relatives were offered an initial face-to-face session in which they were introduced to the toolkit and arrangements for support agreed. Support was offered by email or telephone (relatives' preference) for a maximum of $1 \mathrm{~h}$ per week over 6 months. To ensure communication was maintained, supporters were asked to contact relatives monthly as a minimum if the relatives did not respond to appointments or initiate contact.

Support was targeted at helping relatives to identify the key difficulties they faced and guiding them to find the most relevant information and strategies in the toolkit. Discussion then focused on making these general principles as directly relevant as possible to each individual participant, and on helping relatives to try out new strategies and reflect on the outcomes of this. The toolkit and the support was designed to make the intervention 'enactive' rather than 'symbolic' i.e. it encourages relatives to actively practise new skills, rather than just providing information. ${ }^{20}$

\section{Treatment as usual}

The TAU, as outlined by the operational policies for all participating NHS trusts, included: a carers' assessment and ongoing assessment of need for relatives; development of a shared formulation of individual and family difficulties; emotional support; information about mental health and the mental health system; practical support - finances, accommodation, etc.; links to other support/ services; early crisis intervention; relapse prevention; stress management; referral to structured family intervention where needed. Two of the trusts also ran monthly family and friends peer support groups facilitated by a health professional. Participants in TAU were asked to complete a checklist to indicate to what extent they felt they had received each of these elements. This was used to describe the TAU intervention.

\section{Measures}

This paper reports on the impact of the REACT intervention on the main outcome measures, which were selected for being valid and reliable measures of distress and well-being. The following measures were used.

(a) The General Health Questionnaire (GHQ-28) ${ }^{21}$ was used to assess distress. This is a widely used measure and allowed comparison with other studies in this area. Participants indicate whether their current state differs from their usual state, thereby assessing recent changes in distress as opposed to long-term traits or illnesses. Scoring using the Likert scale method was used for the analysis. We also report on clinical 'caseness' defined as scoring $6+$ using the standard scoring $(0,0,1,1)^{21}$

(b) The Experience of Caregiving Inventory ${ }^{22}$ measures the experience of caring for a relative with a serious mental illness. Totals for the negative and positive subscales were used.

(c) The Carer Well-Being and Support Questionnaire ${ }^{23}$ is designed to cover all aspects of the carer's experience of caring for someone with a serious mental health problem including relationships, roles, financial concerns, physical/ emotional health, stigma, worries about safety, their satisfaction with support offered and ease of obtaining information.

(d) The Family Questionnaire (FQ) ${ }^{24}$ was used to assess relatives' concern and perceived ability to cope. The FQ presents participants with a range of symptoms of mental illness that have the potential to pose a problem in family life. It assesses the extent to which each of these symptoms cause the relative concern and how well the relative is able to cope with their concerns.

(e) The Herth Hope Index ${ }^{25}$ consists of 12 self-report items designed to assess hope in adults in clinical settings.

\section{Quality assurance}

All baseline assessments were carried out by two research assistants who were trained in administering and scoring all measures. Training included role-play practise interviews with relatives and weekly supervision to discuss clinical and scoring 
issues as they arose. Following randomisation, one of the research assistants was made aware of allocation and carried out all communication with participants and associated health professionals and in-depth qualitative interviews with participants during the process of the study. She contacted all participants prior to their follow-up appointment to emphasise the importance of not revealing allocation to the second research assistant at the follow-up interview. The second research assistant remained masked to allocation, was housed in a separate office, had no direct contact with participants or health professionals outside the follow-up interview and no access to databases that contained participant allocation details.

\section{Statistical analysis}

Analyses were conducted on an intention-to-treat basis. A per protocol analysis was not considered appropriate as we deliberately did not specify a protocol for how much time participants should spend reading the toolkit, or how much support they should receive as this should vary according to need. All participants in the intervention arm received the toolkit and offer of support. The two-sided significance level was set at alpha $(\alpha)=0.05$. Where data for an entire measure were missing (i.e. non-response on all items), this was regarded as a missing value. If less than $10 \%$ of items on a single measure were missing, these missing items were imputed by the average of the non-missing items for that participant (i.e. 'prorated').

Comparison between those receiving TAU and those receiving TAU plus REACT on each of the outcomes was carried out using ANCOVA controlling for baseline scores and clinical early intervention services team. Missing data will not invalidate the ANCOVA method as long as the missing data are occurring at random, conditional on the covariates in the model (MAR, using the terminology of Little \& Rubin $^{26}$ ). Regression coefficients with $95 \%$ confidence intervals are reported along with robust standard errors and $P$-value (using the robust option in Stata's regress command (Stata version 11 on Windows 12 was used)). Robust standard errors were used so that the results were not dependent on assumptions of normality.

Chi-squared analyses were used to compare the numbers of relatives moving between the categories of 'caseness' and 'noncaseness' in the two arms to give a more clinical interpretation of the findings.

\section{Results}

\section{Participants - recruitment and retention}

A total of 144 relatives were initially referred into the study (Fig. 1). However, 21 subsequently declined to take part (15 gave no reason, 3 felt they did not have time, 1 disagreed with the diagnosis their relative had been given, and 2 felt they already had enough support). Eight were subsequently identified as ineligible ( 4 had been in the service too long, 1 was unable to understand English sufficiently, 1 was blind, 1 was a service user and 1 was not in the participating early intervention services) and 12 were not contactable. Therefore a total of 103 relatives were randomised.

The majority were female ( 85 female, 18 male), White British $(n=97,94 \%)$ and the mother of the service users $(n=76,74 \%)$. There were $46(45 \%)$ who were unemployed or retired, 34 $(33 \%)$ in full-time work and $22(21 \%)$ in part-time work. In total $86(83 \%)$ were aware of a diagnosis having been given, the most common one being psychosis $(n=43,50 \%)$, schizophrenia $(n=7,8 \%)$ and bipolar disorder $(n=4,5 \%)$. The majority of relatives lived with the service user all of the time $(n=75,73 \%)$. Relatives' perception of how long the service user had experienced

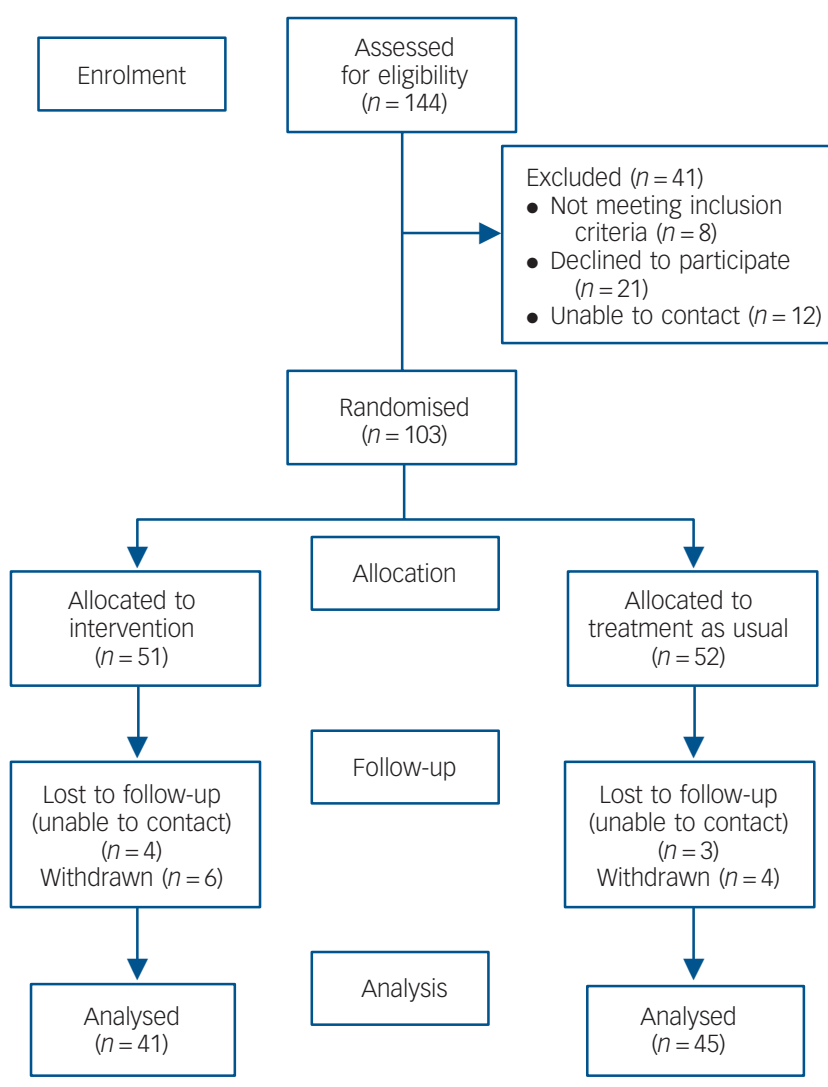

Fig. 1 CONSORT diagram showing flow of participants through the study.

mental health problems was skewed with a median of 36 months (range 3-240) and their perceived time since diagnosis was a median of 9 months (range 0-144). Self-reported length of time in the early intervention services team was reported as a median of 9.5 months (range 2-34.5 months).

\section{Participants' baseline scores}

Baseline scores are shown in Table 1. Levels of distress were generally higher than those reported in previous samples of relatives of people in early psychosis. ${ }^{27,28}$ Sixty-seven (65\%) relatives scored above the threshold for clinical caseness at baseline, suggesting this was a highly distressed group. There was no significant difference between those retained at follow-up and those lost to follow-up on the GHQ at baseline.

Where available, scores on the other measures were also compared with previous studies of comparable samples. Relatives in this study reported equivalent levels of hope, ${ }^{29}$ perceived coping, ${ }^{27}$ carer well-being and support, ${ }^{23}$ and high levels of negative caregiving experiences. ${ }^{3,30,31}$ There are no accepted norms of clinical cut-offs for these measures.

\section{Intervention and support}

All participants allocated to the intervention arm received the REACT toolkit. Forty-seven relatives (92\%) also had a face-to-face introductory session lasting between 35 and $90 \mathrm{~min}$, with a median of $60 \mathrm{~min}$ (interquartile range $(\mathrm{IQR})=45-75)$. The number of subsequent support sessions (defined as contacts lasting $5 \mathrm{~min}$ or longer) ranged from 0 to 17 , with a median number of 3 sessions $(\mathrm{IQR}=2-6)$. The length of support sessions ranged from 5 to $90 \mathrm{~min}$, with a median of $25 \mathrm{~min}$ $(\mathrm{IQR}=15-40)$. Total minutes of REACT supporter contact with 


\begin{tabular}{|c|c|c|c|c|c|}
\hline & $\begin{array}{l}\text { Baseline } \\
\text { Mean (s.d.) }\end{array}$ & $\begin{array}{l}\text { Follow-up } \\
\text { Mean (s.d.) }\end{array}$ & $\begin{array}{l}\text { Treatment effect } \\
\qquad(95 \% \mathrm{Cl})\end{array}$ & $\begin{array}{l}\text { Robust } \\
\text { s.e. }\end{array}$ & $P$ \\
\hline $\begin{array}{l}\text { General Health Questionnaire } \\
\text { REACT team } \\
\text { TAU team }\end{array}$ & $\begin{array}{l}34.21(15.67) \\
34.07(19.00)\end{array}$ & $\begin{array}{l}23.42(15.20) \\
28.30(15.42)\end{array}$ & $-6.59(-12.55$ to -0.64$)$ & 2.99 & 0.030 \\
\hline $\begin{array}{l}\text { Experience of Caregiving Inventory - negative subscale } \\
\text { REACT team } \\
\text { TAU team }\end{array}$ & $\begin{array}{r}95.00(33.94) \\
108.43(35.42)\end{array}$ & $\begin{array}{r}85.53(43.69) \\
100.91(34.93)\end{array}$ & $-9.79(-22.41$ to 2.83$)$ & 6.34 & 0.126 \\
\hline $\begin{array}{l}\text { Experience of Caregiving Inventory - positive subscale } \\
\text { REACT team } \\
\text { TAU team }\end{array}$ & $\begin{array}{l}28.39(8.59) \\
29.68(9.60)\end{array}$ & $\begin{array}{l}30.70(10.56) \\
30.64(9.28)\end{array}$ & $0.87(-2.36$ to 4.09$)$ & 1.62 & 0.593 \\
\hline $\begin{array}{l}\text { Carer Well-being and Support Questionnaire - well-being } \\
\text { REACT team } \\
\text { TAU team }\end{array}$ & $\begin{array}{l}77.91(27.27) \\
70.46(31.44)\end{array}$ & $\begin{array}{l}89.61(29.71) \\
79.50(32.47)\end{array}$ & $9.77(-0.14$ to 19.68$)$ & 4.97 & 0.053 \\
\hline $\begin{array}{l}\text { Carer Well-being and Support Questionnaire - support } \\
\text { REACT team } \\
\text { TAU team }\end{array}$ & $\begin{array}{l}32.72(11.86) \\
28.10(11.05)\end{array}$ & $\begin{array}{l}39.60(10.31) \\
33.89(12.19)\end{array}$ & 4.86 (0.77 to 8.96$)$ & 2.06 & 0.021 \\
\hline $\begin{array}{l}\text { Family Questionnaire - concern } \\
\text { REACT team } \\
\text { TAU team }\end{array}$ & $\begin{array}{l}71.82(12.46) \\
78.19(15.36)\end{array}$ & $\begin{array}{l}68.92(16.95) \\
76.62(16.95)\end{array}$ & -3.12 ( -9.33 to 3.08$)$ & 3.11 & 0.319 \\
\hline $\begin{array}{l}\text { Family Questionnaire - coping } \\
\text { REACT team } \\
\text { TAU team }\end{array}$ & $\begin{array}{l}41.53(19.57) \\
52.71(21.62)\end{array}$ & $\begin{array}{l}40.03 \text { (19.92) } \\
54.25(20.73)\end{array}$ & $-4.89(-9.34$ to -0.44$)$ & 2.24 & 0.032 \\
\hline $\begin{array}{l}\text { Herth Hope Index } \\
\text { REACT team } \\
\text { TAU team }\end{array}$ & $\begin{array}{l}35.32(5.42) \\
36.01(7.01)\end{array}$ & $\begin{array}{l}37.94(5.94) \\
37.15(6.77)\end{array}$ & $1.34(-0.72$ to 3.39$)$ & 1.03 & 0.198 \\
\hline
\end{tabular}

relatives over the 6-month intervention period ranged between 0 and $855 \mathrm{~min}$, with a median of $125.5 \mathrm{~min}$ ( $\mathrm{IQR}=75-204)$.

A total of 31 participants $(61 \%)$ received support via telephone only, $6(12 \%)$ by email only and $7(14 \%)$ had a combination of both. Three participants $(6 \%)$ did not receive any additional support after the introductory session. Only one participant requested access to the online version of the toolkit.

Six NHS supporters were initially trained to support REACT. All had an interest in mental health. One had been a service user and one was a parent of someone with mental health difficulties. Although feedback about the training was generally positive, one dropped out during training as a result of leaving his post in the service, one dropped out following training because of changes in his role within the early intervention team and one was unable to continue because of ill health. Supporters who remained with the study worked flexibly to cover work across teams and additional input was required from one of the research team, a trained nurse therapist (D.G.) to support three participants.

\section{Treatment as usual}

Participants in the TAU group were asked to give an overall rating of how satisfied they were with the amount of help and support they had received from early intervention services. Forty-four people completed this measure. Of these, $14(32 \%)$ were very satisfied, $14(32 \%)$ were mostly satisfied, 4 (9\%) felt indifferent, $9(21 \%)$ were only moderately satisfied and $3(7 \%)$ were not at all satisfied. Table 2 shows participants' ratings of perceived support for each of the key elements of TAU. Deficits were most apparent in practical support, for example regarding finances and accommodation, and advice regarding stress management, for which at least half the sample did not feel they had received any support.

\section{Impact on main outcomes}

The estimated intervention effects (regression coefficient) shown in Table 1 indicate that relatives who received REACT showed a greater reduction in distress, negative experiences of caregiving, and concern about psychosis, along with increased positive experiences of caregiving, carer well-being and support, and perceived ability to cope (a high score indicates less coping) compared with those in the TAU group. However, only reduction in distress, increase in sense of being supported and increase in perceived coping were statistically significant.

In the REACT group 14 of the 27 relatives (52\%) categorised as clinical cases at baseline no longer reached this threshold compared with 7 of the 25 relatives (28\%) in the TAU group. This difference just failed to reach statistical significance (Pearson's $\chi^{2}=3.07, P=0.08$ ). No differences were found between groups for those who did not meet criteria for clinical caseness at baseline with $21 \%$ of these participants meeting the threshold at follow-up in both arms. This suggests that REACT may be of clinical value to those scoring above threshold for clinical caseness but there is no evidence of a positive or negative impact on those scoring below, although much larger studies are needed to test this directly.

\section{Quality assurance}

Despite attempts to maintain masking, the second research assistant was unmasked to the allocation of nine participants as a result of the relative having the toolkit visibly present at the interview or referring directly to the support they had received. Full masking was maintained for the remaining 77 participants.

\section{Discussion}

\section{Main findings and comparison with other studies}

This study provides preliminary evidence for the feasibility and effectiveness of a supported self-management intervention for relatives of people experiencing recent-onset psychosis in a realworld clinical setting. Compared with similar studies recruiting 
Table 2 Relatives' report of what support they had received in the treatment as usual (TAU) group

\begin{tabular}{|c|c|c|c|c|}
\hline \multirow[b]{2}{*}{ Checklist to assess content of TAU } & \multicolumn{4}{|c|}{ Frequency (\% of total sample) $(n=52)$} \\
\hline & Fully & Somewhat & Not at all & Missing \\
\hline $\begin{array}{l}\text { 1. Do you think your needs as a carer/family member were assessed by the early intervention } \\
\text { services team? }\end{array}$ & $16(31)$ & $24(46)$ & $4(8)$ & $8(15)$ \\
\hline 2. Were any problems that you were having identified? & $15(29)$ & $23(44)$ & $6(12)$ & $8(15)$ \\
\hline 3. Did you receive emotional support from the early intervention team? & $10(19)$ & $24(46)$ & $10(19)$ & $8(15)$ \\
\hline 4. Did you receive information about the mental health system? & $17(33)$ & $21(40)$ & $6(12)$ & $8(15)$ \\
\hline 5. Did you receive practical support, for example regarding finances, accommodation, etc? & 7 (14) & $15(29)$ & $22(42)$ & $8(15)$ \\
\hline $\begin{array}{l}\text { 6. Were you informed about other services that may be able to help you, for example charitable } \\
\text { organisations such as a local example given? }\end{array}$ & $6(12)$ & $20(39)$ & $18(35)$ & $8(15)$ \\
\hline $\begin{array}{l}\text { 7. Do you have contact details of someone that you can contact if your relative is experiencing } \\
\text { a crisis: } 8 \text { am to } 8 \mathrm{pm} \text { ? }\end{array}$ & $32(62)$ & $8(15)$ & $4(8)$ & $8(15)$ \\
\hline $\begin{array}{l}\text { 8. Do you have contact details of someone that you can contact if your relative is experiencing } \\
\text { a crisis: out of hours? }\end{array}$ & $30(58)$ & $6(12)$ & $7(14)$ & $9(17)$ \\
\hline 9. Were you given any information regarding the nature of your relative's mental health problems? & $16(31)$ & $24(46)$ & $4(8)$ & $8(15)$ \\
\hline 10. Were you given advice regarding stress management? & $6(12)$ & $15(29)$ & $22(42)$ & $9(17)$ \\
\hline 11. Were you given information regarding relapse prevention? & $13(25)$ & $13(25)$ & $18(35)$ & $8(15)$ \\
\hline $\begin{array}{l}\text { 12. Was an assessment made of any possible risks of harm to your relative or someone else } \\
\text { and a strategy agreed to manage these? }\end{array}$ & $15(29)$ & $12(23)$ & $16(31)$ & $9(17)$ \\
\hline
\end{tabular}

families of people with psychotic disorders, relatives were keen to take part in the study, and follow-up rates were high. ${ }^{30,32,33}$ Relatives showed high levels of distress ${ }^{27,28}$ and negative experiences of caregiving $^{3,30}$ compared with previous studies with similar samples.

Compared with TAU only, those receiving the additional REACT intervention showed significant positive changes on a range of outcome measures including reduced distress, increased support and increased perceived ability to cope. A trend was found for increased well-being $(P=0.053)$. There were no significant differences between the groups on negative or positive experiences of caregiving, concern about service-user behaviour, or hope, although all the non-significant differences observed were in the direction favouring a more positive change for those receiving REACT.

These positive findings are consistent with evidence for the effectiveness of supported self-management interventions to reduce distress in relatives of people with eating disorders ${ }^{15,16}$ but are perhaps surprising given that the relatives in this study are already in contact with early intervention service teams offering a very comprehensive package of support for relatives. Despite having been with the teams for many months, the relatives were still benefitting from this additional supported self-management approach. Relatives' self-report of TAU suggests that this may be because of difficulties in delivery of family support protocols as many did not feel they were receiving the interventions specified in the NHS trust policies. Self-management toolkits such as REACT have the potential to ensure some of these key elements, such as practical advice about finance and accommodation, signposting to other relevant services and advice on stress management, can be readily available.

The toolkit was supported by existing NHS staff. There were challenges in retaining trained supporters to the study, and future use of the toolkit may benefit from training more than one team member to accommodate sick leave and staff turnover. There was a strong preference by relatives for a printed version of the toolkit and a moderate preference for support by telephone. This was surprising given the recent explosion of internet use and the success of online support for relatives in other areas ${ }^{16}$ and may be partly the result of the recruitment strategy (which was via face-to-face contact, rather than online) or a lack of promotion of the online options by supporters who were less familiar with working this way.
The amount of support relatives received also varied considerably. This study is too small to examine quantitatively the role of the support and whether there is a minimum amount of support required for the toolkit to be effective. Indeed, it is possible that the support rather than the toolkit is the essential component, although research in eating disorders would suggest this is not the case. ${ }^{15}$

\section{Study limitations}

The study is a small feasibility trial. The findings suggest the REACT intervention can be effective in improving key outcomes for relatives but large confidence intervals indicate the need for replication in a larger trial to assess their reliability. This study focused on an intervention for relatives aimed at improving outcomes for relatives. The impact on service user outcomes was not assessed, and no service user characteristics were controlled for. Based on interpersonal models of caregiving, ${ }^{34}$ we would hypothesise that improving outcome for relatives will have a positive impact on service user outcomes, but further studies are needed to test this. However, this would require service users' willing participation, excluding relatives who may be most in need of this kind of support. A key strength of REACT is that it is available to all relatives.

There was no attempt to assess the cost-effectiveness of the intervention at this stage. The REACT intervention is designed to be a low-intensity intervention that can be made widely available to all relatives. It therefore has the limitations of being generic and limited in scope. However, it is not intended to replace high-intensity structured family interventions, which can offer a tailored formulation-driven approach and should continue to be offered to families that require this level of input. The toolkit was offered as an adjunct to TAU, however TAU was not assessed in the REACT arm. It is possible that TAU varied between the arms, which could bias the results.

\section{Clinical implications}

There is a worldwide recognition of the need to develop new ways to disseminate psychological therapies in order to meet growing demand. ${ }^{35}$ This toolkit offers a low-intensity intervention for relatives of people with recent-onset psychosis that has the 
potential to increase access to the key components of existing evidence-based interventions. There is scope for future development of the REACT toolkit to include: peer support, interactive online programmes and adaptation to the specific needs of relatives such as those from minority ethnic groups, young family members and siblings. The modular design of the toolkit format also lends itself to adaption to other clinical problems such as bipolar disorder.

\section{Future research}

A larger trial is needed to reliably assess the clinical and costeffectiveness of the REACT intervention. This study should focus on long- as well as short-term outcomes and further attempt to identify the contribution of the toolkit and support in determining outcome. If the intervention is effective, subsequent research should focus on the most effective way to disseminate the intervention, and appropriate adaptations for relatives of people with different diagnoses or from different ethnic or cultural groups. Identifying the optimal time to offer this kind of intervention to relatives is also important. It could be hypothesised that the earlier relatives receive this information, the more effective it may be as it ensures access to information when it is needed. However, it may be that for some relatives too much information too soon can feel overwhelming and lead to it being ignored or even increasing levels of distress. Establishing mechanisms of action and treatment moderators would help identify the key components to aid further development and dissemination.

Fiona Lobban, BA, DClinPsy, PhD, Spectrum Centre, Division of Health Research, Lancaster University; David Glentworth, RMN, DipCogTher, Bolton Early Intervention Service, Greater Manchester West Mental Health NHS Foundation Trust; Lesley Chapman, relative; Laura Wainwright, BSC, MSC, Adam Postlethwaite, BSC, MSC, Chapman, relative; Laura Wainwright, BSC, MSC, Adam Postlethwaite, BSC, MSC,
Spectrum Centre, Division of Health Research, Lancaster University; Graham Dunn $\mathrm{BA}, \mathrm{MA}, \mathrm{PhD}, \mathrm{MSC}$, Centre for Biostatistics, University of Manchester; Vanessa Pinfold, BA, PhD, Institute of Psychiatry, London; Warren Larkin, BSC, DClinPsy, Children and Families Network, Lancashire Care NHS Foundation Trust, Preston; Gillian Haddock, BSC, MSC, PhD, School of Psychological Sciences, University of Manchester, UK

Correspondence: Fiona Lobban, Spectrum Centre, Institute for Health Research, Lancaster University, Lancaster LA1 4YT, UK. Email: f.lobban@lancaster.ac.uk

First received 22 Apr 2012, final revision 11 Apr 2013, accepted 8 May 2013

\section{Funding}

This paper presents independent research commissioned by the National Institute for Health Research (NIHR) under its Research for Patient Benefit (RfPB) Programme (Grant Reference Number RfPB PB-PG-0807-14075).

\section{Acknowledgements}

We would like to acknowledge the kind support of the trial steering committee chaired by Professor Karina Lovell, and including Dr Chris Sutton, Dr Bill Sellwood and Ms Ros Bentley. We would like to thank all the relatives who participated in this study.

\section{References}

1 Winefield HR, Harvey EJ. Determinates of psychological distress in relatives of people with chronic schizophrenia. Schizophr Bull 1993; 19: 619-25.

2 Barrowclough $\mathrm{C}$, Tarrier N, Johnston $\mathrm{M}$. Distress, expressed emotion, and attributions in relatives of schizophrenia patients. Schizophr Bull 1996; 22 : 691-702.

3 Addington J. An integrated treatment approach to substance use in an early psychosis programme. In Substance Misuse in Psychosis: Approaches to Treatment and Service Delivery (eds HL Graham, A Copello, MJ Birchwood, KT Mueser): 121-35. John Wiley \& Sons, 2003.

4 Barrowclough C, Haddock G, Beardmore R, Conrod P, Craig T, Davies L, et al. Evaluating integrated $\mathrm{MI}$ and $\mathrm{CBT}$ for people with psychosis and substance misuse: recruitment, retention and sample charateristics of the MIDAS trial. Addict Behav 2009; 34: 859-66.

5 Department of Health. Carers at the Heart of 21st-Century Families and Communities. Department of Health, 2008.

6 Buckner L, Yeandle S. Valuing Carers 2011: Calculating the Value of Carers' Support. Carers UK, 2011.

7 Pharoah FM, Rathbone J, Mari JJ, Streiner D. Family intervention for schizophrenia. Cochrane Database Syst Rev 2006; 4: 112.

8 National Institute for Health and Clinical Excellence. Core Interventions in the Treatment and Management of Schizophrenia in Adults in Primary and Secondary Care (Update). NICE, 2009.

9 Bird V, Premkumar P, Kendall T, Whittington C, Mitchell J, Kuipers E. Early intervention services, cognitive-behavioural therapy and family intervention in early psychosis: systematic review. Br J Psychiatry 2010; 197: 350-6.

10 Linszen D, Dingemans P, Van der Does JW, Nugter A, Scholte P, Lenior R, et al. Treatment, expressed emotion and relapse in recent onset schizophrenic disorders. Psychol Med 1996; 26: 333-42.

11 Lobban F, Postlethwaite A, Glentworth D, Pinfold V, Wainwright L, Dunn G, et al. A systematic review of randomised controlled trials of interventions reporting outcomes for relatives of people with psychosis. Clin Psychol Rev 2013; 33: 372-82.

12 Fadden G. Training and disseminating family interventions for schizophrenia: developing family intervention skills with multi-disciplinary groups. J Fam Ther 2006; 28: 23-38.

13 Department of Health. Supporting People with Long-Term Conditions: An NHS and Social Care Model to Support Local Innovation and Integration. Department of Health, 2005.

14 Barlow J, Wright C, Sheasby J, Turner A, Hainsworth J. Self-management approaches for people with chronic conditions: a review. Patient Educ Couns 2002; 48: 177-87.

15 Goddard E, Macdonald P, Sepulveda AR, Naumann U, Landau S, Schmidt U, et al. Cognitive interpersonal maintenance model of eating disorders: intervention for carers. Br J Psychiatry 2011; 199: 225-31.

16 Grover M, Naumann U, Mohammad-Dar L, Glennon D, Ringwood S, Eisler I, et al. A randomized controlled trial of an internet-based cognitivebehavioural skills package for carers of people with anorexia nervosa. Psychol Med 2011; 41: 2581-91.

17 Lobban F, Glentworth D, Wainwright L, Pinfold V, Chapman I, Larkin W, et al. Relatives Education And Coping Toolkit - REACT. Study protocol of a randomised controlled trial to assess the feasibility and effectiveness of a supported self management package for relatives of people with recent onset psychosis. BMC Psychiatry 2011; 11: 100.

18 Department of Health. The Mental Health Policy Implementation Guide. Department of Health, 2001.

19 Lobban F, Glentworth D, Haddock G, Wainwright L, Clancy A, Bentley R, et al. The views of relatives of young people with psychosis on how to design a Relatives Education And Coping Toolkit (REACT). J Ment Health 2011; 20 : 567-79.

20 Barrowclough C, Tarrier N. 'Psychosocial' interventions with families and their effects on the course of schizophrenia: a review. Psychol Med 1984; 14: 629-42.

21 Goldberg DP, Hillier VF. Scaled version of the General Health Questionnaire. Psychol Med 1979; 9: 139-45.

22 Szmukler Gl, Burgess P, Herrman H, Bloch S, Benson A, Colusa S. Caring for relatives with serious mental illness: the development of the Experience of Caregiving Inventory. Soc Psychiatry Psychiatr Epidemiol 1996; 31: 137-48.

23 Quirk A, Smith SC, Hamilton S, Lamping DL, Lelliot P, Stahl D, et al. Development and Validation of the Carer Well-Being and Support (CWS) Questionnaire: Report for the National Institute for Health Research Service Delivery and Organisation Programme. HMSO, 2009 (http:// www.netscc.ac.uk/hsdr/files/project/SDO_FR_08-1613-144_V01.pdf).

24 Quinn J, Barrowclough C, Tarrier N. The Family Questionnaire (FQ): a scale for measuring symptom appraisal in relatives of schizophrenic patients. Acta Psychiatr Scand 2003; 108: 290-6.

25 Herth K. Abbreviated instrument to measure hope - development and psychometric evaluation. J Adv Nurs 1992; 17: 1251-9.

26 Little RJA, Rubin DR. Statistical Analysis with Missing Data (2nd edn). Wiley, 2002.

27 Barrowclough C, Gooding P, Hartley S, Lee G, Lobban F. Factors associated with distress in relatives experiencing recent onset psychosis in a family member. J Nerv Ment Dis 2013; in press.

28 Szmukler $\mathrm{Gl}$, Herrman $\mathrm{H}$, Colusa S, Benson A, Bloch S. A controlled trial of a counselling intervention for caregivers of relatives with schizophrenia. SoC Psychiatry Psychiatr Epidemiol 1996; 31: 149-55. 
29 Benzein EG, Berg AC. The level of and relation between hope, hopelessness and fatigue in patients and family members in palliative care. Palliat Med 2005; 19: 234-40.

30 Szmukler G, Kuipers E, Joyce J, Harris T, Leese M, Maphosa W, et al. An exploratory randomised controlled trial of a support programme for carers of patients with a psychosis. Soc Psychiatry Psychiatr Epidemiol 2003; 38: $411-8$.

31 Treasure J, Murphy T, Szmukler G, Todd G, Gavan K, Joyce J. The experience of caregiving for severe mental illness: a comparison between anorexia nervosa and psychosis. Soc Psychiatry Psychiatr Epidemiol 2001; 36: 343-7.
32 Barrowclough C, Tarrier N, Lewis S, Sellwood W, Mainwaring J, Quinn J, et al. Randomised controlled effectiveness trial of a needs-based psychosocia intervention service for carers of people with schizophrenia. Br J Psychiatry 1999; 174: 505-11.

33 Leavey G, Gulamhussein S, Papadopoulos C, Johnson-Sabine E, Blizard B, King M. A randomized controlled trial of a brief intervention for families of patients with a first episode of psychosis. Psychol Med 2004; 34: 423-31.

34 Kuipers E, Onwumere J, Bebbington P. Cognitive model of caregiving in psychosis. Br J Psychiatry 2010; 196: 259-65.

35 Kazdin AE, Blasé SL. Rebooting psychotherapy research and practice to reduce the burden of mental illness. Perspect Psychol Sci 2011; 6: 21-37.

\section{Cornelius Katona}

This radical volume appeared while I was an SHO. My main mentors at the time were Sir Martin Roth and German Berrios. Aliens and Alienists provided a very different, and quite heady, perspective for an easily influenced young trainee like me, who saw himself working steadily towards a distant goal of biological erudition. Long before Sir Robin Murray's group's seminal work on the complex links between cannabis and schizophrenia, it provided a measured discussion of the inter-relationships between Rastafarianism, cannabis, the experience of immigration and racial abuse, and the development of disturbed behaviour. More fundamentally, it encouraged psychiatrists to listen carefully to individual patients and to think about how their behaviour might reflect their past experiences, as well as being a manifestation of a genetic or neurochemical abnormality. This was of course fully in keeping with the post-Kraepelinian emphasis on phenomenology which was the common ground for Roth and Berrios and which made the discussions at their joint ward rounds so enthralling

As I re-read my well-thumbed and somewhat foxed blue-spined Penguin edition of Aliens and Alienists in preparation for writing this reflection, I was reminded of a particular patient I saw at about that time. She was a member of a charismatic church, and had been admitted in a very excitable state, seemed grandiose and disinhibited and, most importantly, speaking in tongues. Was she manic or was this acceptable behaviour within her religious grouping? After a lot of discussion within the multidisciplinary team, it was decided that I should speak to her Church pastor. He came and visited, and within a few minutes said with great confidence that she was severely ill. She responded well to treatment for her bipolar disorder while retaining her faith and her occasional ability to speak in tongues.

I was privileged to work with one of the book's authors, Roland Littlewood. Unlike the rest of us he was a real academic, whose research consisted of field work in faraway countries rather than the completion of rating scales and taking of blood samples that preoccupied the rest of us. But the main memory I retain of Roland's contribution is his remarkable storytelling skills, which brought to life societies and individuals very different to those I saw in clinical practice in Essex. It is this storytelling which is, to my mind, the most enduring aspect of Aliens and Alienists. The case vignettes Littlewood and Lipsedge provide are short, but they are as vivid as Alice Munro short stories and provide a vivid underpinning to what would otherwise be quite dry social and anthropological analysis.

My own psychiatric journey has not been entirely straightforward. My commitment to the subject stemmed from a fascination with Freudian analysis from my university years, but my early research was purely biological. I spent most of my career in old age psychiatry and have now found a new 'home' working with asylum seekers who have survived torture and other human rights abuses. It is in this new home at the Helen Bamber Foundation (www.helenbamber.org) that the relevance of Aliens and Alienists has been brought home to me most forcefully. One of the most important tasks is, in Helen Bamber's words, to 'bear witness', to listen to the survivor's story. The perspective Littlewood and Lipsedge provide, with its emphasis on imagining and recreating the lived experience of people, has proved invaluable in helping me to give them space to tell their story, and to try not to impose my own cultural perspective on what they say. 